\title{
HIGHLY EFFICIENT THIRD HARMONIC GENERATION BY MEANS OF FOUR-WAVE DIFFERENCE-FREQUENCY MIXING IN FUSED SILICA
}

\author{
J. Darginavičius, D. Majus, G. Tamošauskas, and A. Dubietis \\ Department of Quantum Electronics, Vilnius University, Sauletekio 9, LT-10222 Vilnius, Lithuania \\ E-mail: audrius.dubietis@ ff.vu.lt
}

Received 29 January 2009; accepted 19 March 2009

\begin{abstract}
We report on highly efficient third harmonic generation of 1-ps laser pulses in transparent isotropic solid state medium through phase-matched non-collinear four-wave difference-frequency mixing. Third harmonic pulses at $351 \mathrm{~nm}$ with $230 \mu \mathrm{J}$ energy and 15\% energy conversion efficiency were generated in 3-mm-thick UV-grade fused silica sample by use of noncollinear phase matching, cylindrical beam focusing geometry, and pulse-front tilting.
\end{abstract}

Keywords: third harmonic generation, four-wave mixing

PACS: $42.65 . \mathrm{Ky}, 42.79 . \mathrm{Nv}$

\section{Introduction}

Third harmonic (TH) generation in isotropic media is widely used for femtosecond pulse frequency conversion in the ultraviolet spectral range. In a medium with $\chi^{(3)}$ nonlinearity there are two four-wave mixing (FWM) configurations, which may lead to generation of radiation at $3 \omega$. The first approach considers direct frequency tripling, that is $3 \omega=\omega+\omega+\omega$. The phase matching condition could be fulfilled only in media with anomalous dispersion, e.g. metal vapours [1], however the overall process is inefficient due to high absorption. On the other hand, in transparent media with normal dispersion, the phase-mismatch could be greatly reduced by means of tight beam focusing and occurrence of non-perturbative effects at high intensity limit $[2,3]$. In this case, however, energy conversion process is limited by low intrinsic nonlinearity of gaseous media and short interaction length. The efficiency of TH generation could be improved by use of high pressure gases [4] or by the guided wave propagation in the filamentation regime [3,5], but still does not exceed $1 \%$. In condensed media, $\mathrm{TH}$ generation efficiency in the filamentation regime still remains remarkably low $\left(10^{-6}\right)$ due to severe phase-matching limitations [6]. The second approach is based on the fourwave difference-frequency mixing $(3 \omega=2 \omega+2 \omega-\omega)$ and is more flexible in terms of the interaction geometry. Using this approach, femtosecond $\mathrm{TH}$ pulses were generated in a noble-gas-filled capillary waveguide [7] and in an extended light filament generated in noble gasses at atmospheric pressure [8] with improved energy conversion efficiency of $2-4 \%$. The use of cascaded four-wave mixing processes enables further frequency conversion to fourth and fifth laser harmonics [9]. The perfect phase matching may be achieved by setting a non-collinear interaction geometry, for instance, by coupling of Bessel and Gaussian beams at fundamental and second harmonics, respectively [10]. In a condensed medium, enhanced TH generation in water by the use of elliptical light beams was reported [11].

The limiting factor for efficient $\mathrm{TH}$ generation in a solid state media is the optical damage. Therefore cylindrical beam focusing enables to scale the energy of the interacting beams keeping the overall fluence below the damage threshold, as demonstrated in recent four-wave parametric amplification experiments [1214]. In this paper we report on highly efficient TH generation in fused silica with milijoule pumping, using non-collinear phase matching, cylindrical beam focusing, and pulse-front tilting of one of the pump pulses. 


\section{Experimental results}

The experiment was performed with a $\mathrm{Nd}$ :glass laser system (Twinkle, Light Conversion Ltd.), which delivered 1-ps, 1054-nm pulses at $10 \mathrm{~Hz}$ repetition rate. The laser output was split into two parts. The first portion of the laser radiation was frequency doubled and then recombined with a fresh fundamental harmonic so as to produce $\mathrm{TH}$ through the four-wave differencefrequency mixing process, i. e. $3 \omega=2 \omega+2 \omega-\omega$. Copolarized pump beams (fundamental and second harmonics) were focused into the UV-grade fused silica sample (KU-1) using cylindrical lenses of $f_{x}=\infty$, $f_{y}=+500 \mathrm{~mm}$ and $f_{x}=\infty, f_{y}=+750 \mathrm{~mm}$ respectively, so as to achieve mode matching. The FWHM size of both pump beams on the input face of the fused silica sample was measured as $5 \mathrm{~mm} \times 50 \mu \mathrm{m}$. The fundamental and second harmonic beams were crossed in the plane containing the major axes of the ellipses in order to ensure good spatial overlap along the entire sample length. The crossing angle was found from the phase-matching condition, which is described by a vector equation $\mathbf{k}_{3 \omega}=2 \mathbf{k}_{2 \omega}-\mathbf{k}_{\omega}$, where $|\mathbf{k}(w)|=$ $n(w) w / c$ is the wave number. After simple algebra, one finds the (internal) phase matching angle $\theta$ between the fundamental and second harmonic beams:

$$
\cos \theta=\frac{4 k_{2 \omega}^{2}+k_{\omega}^{2}-k_{3 \omega}^{2}}{4 k_{\omega} k_{2 \omega}},
$$

which yields $\theta=11^{\circ}$ under our particular experimental settings.
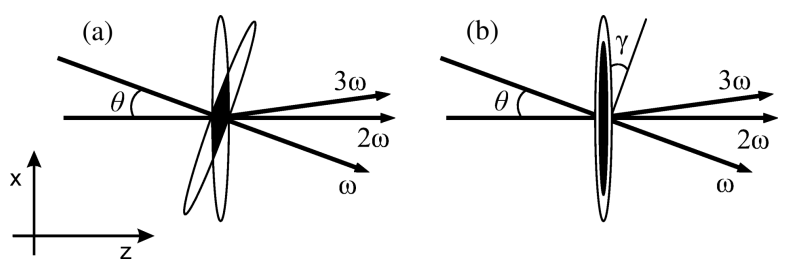

Fig. 1. Schematic representation of pump pulse overlap (indicated by black area) with (a) untilted pulses, (b) tilted-front fundamental harmonic pulse.

Since the elliptical pump beams carry ultrashort light pulses (1-ps pulse occupies only a narrow width of $\approx 200 \mu \mathrm{m}$ along the $z$ axis in fused silica), large beam crossing angle results in noticeable reduction of the beam/pulse physical overlap area in the nonlinear medium, as illustrated in Fig. 1(a), which in turn greatly reduces the dimension of the generated $\mathrm{TH}$ beam along the $x$ axis as shown in Fig. 2. We have improved the overlap geometry by introducing the pulse-front tilt on the fundamental harmonic pulse, as illustrated in
Fig. 1(b). The pulse-front tilt angle $\gamma=8.6^{\circ}$ was produced by means of accomplishing four passes through the TF5 glass prism with an apex angle of $60^{\circ}$ at the minimum deviation geometry. Further we refer to the above configurations as untilted and tilted pulse cases, respectively. The effect of increased spatial overlap due to pulse-front tilt on the TH beam dimension is illustrated in Fig. 2, where TH beam intensity profiles in the case of untilted and tilted pulses are compared. Improvement of the interaction geometry via pulse-front tilting has led to a substantial improvement of the $\mathrm{TH}$ pulse energy as well.

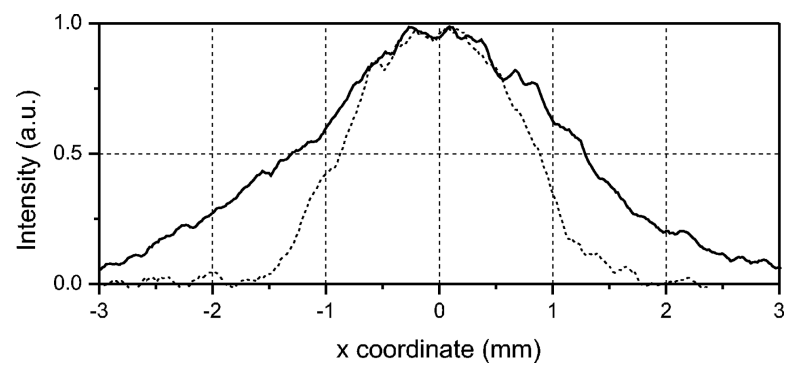

Fig. 2. Measured central cross-sections of TH beams along $x$ axis in the case of untilted (dashed curve) and tilted (solid curve) pulses.

Figure 3 compares the TH pulse energy dependence measured versus pump energy $E_{\text {pump }}$ with untilted and tilted pulses in 3-mm-thick fused silica sample. The pump energy here is defined as $E_{\text {pump }}=E_{\omega}+E_{2 \omega}$, with fixed energy ratio between the pump pulses that was set at $E_{\omega} / E_{2 \omega}=1 / 4$ according to Manley-Rowe relation. The highest value of applicable $E_{\text {pump }}$ was limited by the threshold for the optical damage of fused silica. With tilted pulses, the highest $\mathrm{TH}$ pulse energy of $E_{3 \omega}=230 \mu \mathrm{J}$ is measured at $E_{\text {pump }}=1.55 \mathrm{~mJ}$, suggesting as high as $15 \%$ energy conversion efficiency from the pump. Besides the improvement in the interaction geometry, tilting of one of the pulses leads to a better group velocity matching along the propagation axis ( $z$ axis) for non-collinearly propagating ultrashort pump pulses, which might be important for frequency conversion using femtosecond light pulses.

Figure 4 plots the energy conversion efficiency, $\eta=$ $E_{3 \omega} /\left(E_{\omega}+E_{2 \omega}\right)$, versus pump energy, measured in fused silica samples of different thickness $(3,5$, and $8 \mathrm{~mm}$ ). The measured curves indicate that the TH conversion efficiency saturates at nearly the same $\eta \approx 15 \%$ in all samples, however, at different pump energy as a result of highly nonlinear propagation dynamics.

And finally, we have measured the focusability of the TH beam generated in 3-mm-thick fused silica sample. For many practical applications the beams with circular 


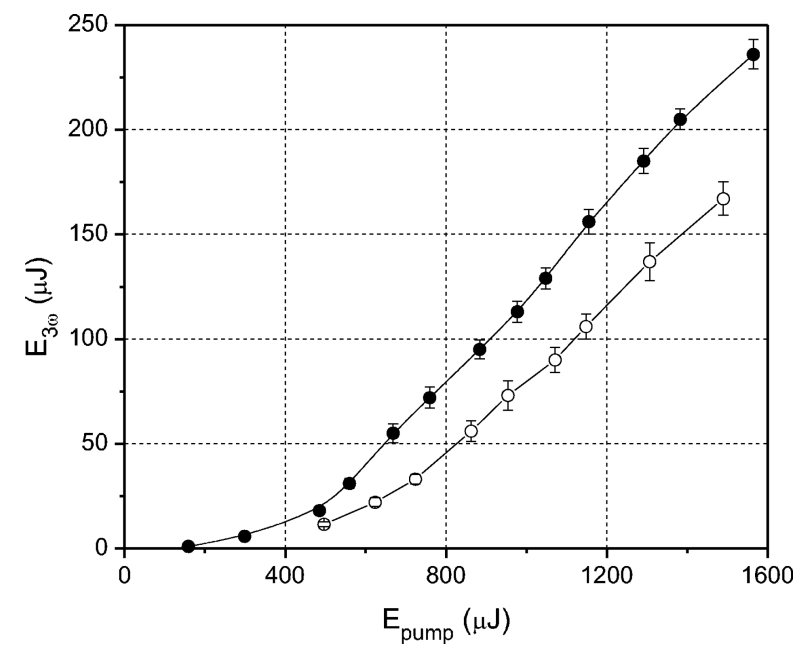

Fig. 3. Third harmonic pulse energy $E_{3 \omega}$ versus pump energy $E_{\text {pump }}$ measured in 3-mm-thick fused silica sample with untilted (open circles) and tilted (solid circles) pulses.

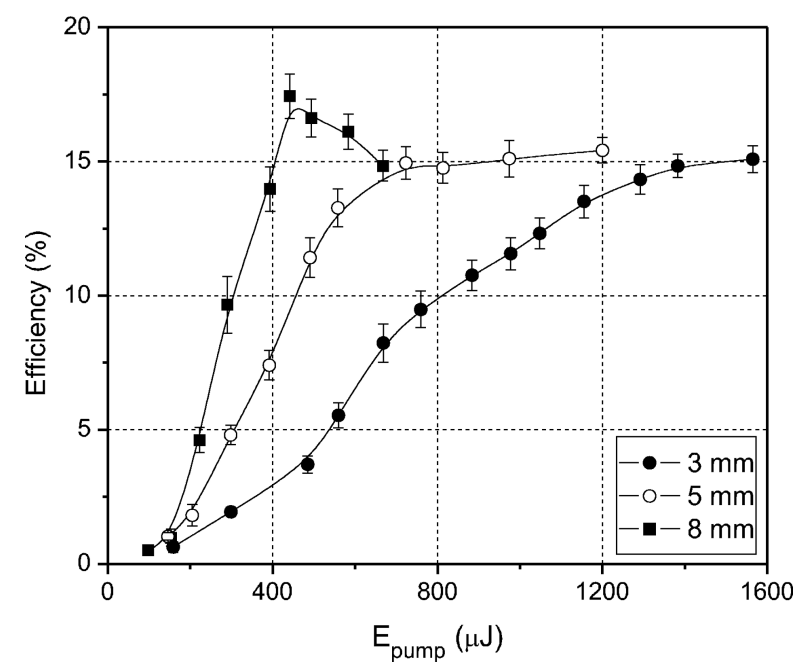

Fig. 4. TH energy conversion efficiency versus pump energy measured in 3,5 , and $8 \mathrm{~mm}$ thick fused silica samples.

symmetry are required, therefore the circular symmetry of the elliptical TH beam was restored using $f_{y}=$ $+100 \mathrm{~mm}$ cylindrical lens. Figure 5 shows the measured TH beam intensity profile and its relevant crosssections at the focal plane of $f=+1 \mathrm{~m}$ spherical lens. The focused TH beam exhibits good focusability and the estimated beam quality is $\approx 1.5$ times of the diffraction limited Gaussian beam.

\section{Conclusion}

In conclusion, we have experimentally demonstrated efficient third harmonic generation of 1-ps Nd:glass laser pulses through four-wave difference-frequency mixing in fused silica with milijoule pumping. TH pulses with energy as high as $230 \mu \mathrm{J}$ with $15 \%$ en-

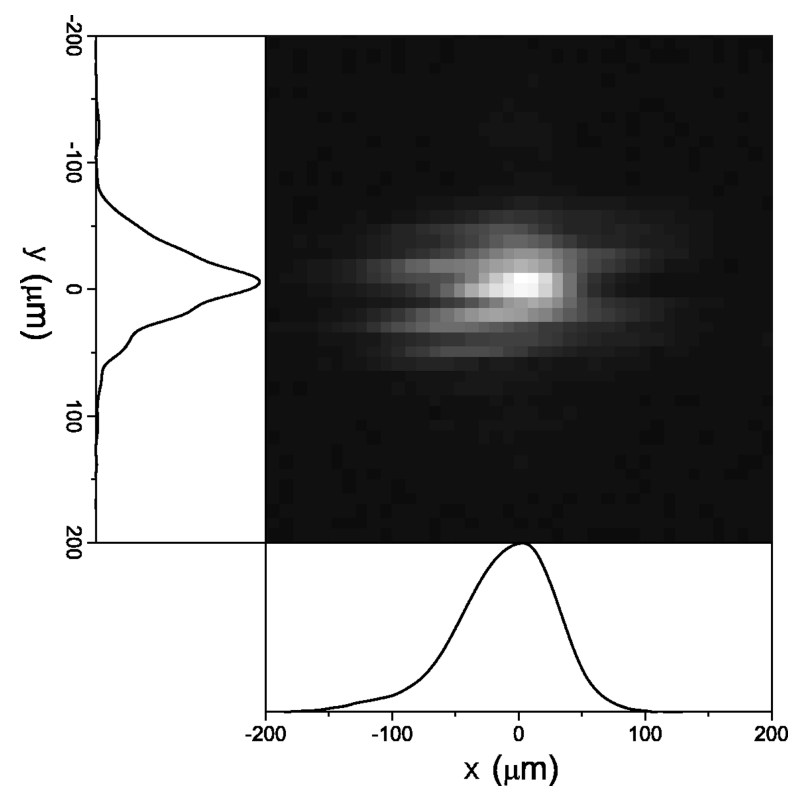

Fig. 5. Intensity distribution of the TH beam and its central crosssections along the principal axes at the focal plane of an $f=+1 \mathrm{~m}$ spherical lens.

ergy conversion efficiency were obtained using noncollinear phase matching combined with cylindrical beam focusing geometry. We also have shown that under these geometrical settings, the efficiency of the TH generation process is notably improved by suitable pulse-front tilting of one of the pump pulses. The proposed set-up could be readily implemented for the frequency conversion of the femtosecond light pulses and for the generation of higher order harmonics in deepUV and VUV-transparent solids, in particular.

\section{Acknowledgement}

Authors acknowledge the financial support from the Lithuanian State Science and Studies Foundation (project FORTAS, No. B-29/2008).

\section{References}

[1] S.E. Harris, and R.B. Miles, Proposed third-harmonic generation in phase-matched metal vapors, Appl. Phys. Lett. 27, 385 (1971).

[2] G.C. Bjorklund, Effects of focusing on third-order nonlinear processes in isotropic media, IEEE J. Quantum Electron. 11, 287-296 (1975).

[3] S. Backus, J. Peatross, Z. Zeek, A. Rundquist, G. Taft, M.M. Murnane, and H.C. Kapteyn, 16-fs, 1- $\mu$ J ultraviolet pulses generated by third-harmonic conversion in air, Opt. Lett. 21, 665-667 (1996).

[4] G. Marcus, A. Zigler, and Z. Henis, Third-harmonic generation at atmospheric pressure in methane by use 
of intense femtosecond pulses in the tight-focusing limit, J. Opt. Soc. Am. B 16, 792-800 (1999).

[5] N. Aközbek, A. Iwasaki, A. Becker, M. Scalora, S.L. Chin, and C.M. Bowden, Third-harmonic generation and self-channeling in air using high-power femtosecond laser pulses, Phys. Rev. Lett. 89, 142901 (2002).

[6] G. Mao, Y. Wu, and K.D. Singer, Third harmonic generation in self-focused filaments in liquids, Opt. Express 15, 4857-4862 (2007).

[7] C.G. Durfee III, S. Backus, M.M. Murnane, and H.C. Kapteyn, Ultrabroadband phase-matched optical parametric generation in the ultraviolet by use of guided waves, Opt. Lett. 22, 1565-1567 (1997).

[8] T. Fuji, T. Horio, and T. Suzuki, Generation of $12 \mathrm{fs}$ deep-ultraviolet pulses by four-wave mixing through filamentation in neon gas, Opt. Lett. 32, 2481-2483 (2007).

[9] L. Misoguti, S. Backus, C.G. Durfee, R. Bartels, M.M. Murnane, and H.C. Kapteyn, Generation of broadband VUV light using third-order cascaded processes, Phys. Rev. Lett. 87, 013601 (2001).
[10] V. Vaičaitis, Cherenkov-type phase-matched third harmonic generation in air, Opt. Commun. 185, 197-202 (2000).

[11] A. Penzkofer, J. Kraus, and J. Sperka, Noncollinear phase matched four photon frequency mixing in water, Opt. Commun. 37, 437-441 (1981).

[12] H. Valtna, A. Dubietis, G. Tamošauskas, P. Polesana, J. Galinis, D. Majus, G. Valiulis, D. Faccio, P. Di Trapani, and A. Piskarskas, Efficient four-wave parametric amplification and spatial soliton generation in transparent isotropic medium with Kerr nonlinearity, Lithuanian J. Phys. 47, 403-410 (2007).

[13] A. Dubietis, G. Tamošauskas, P. Polesana, G. Valiulis, H. Valtna, D. Faccio, P. Di Trapani, and A. Piskarskas, Highly efficient four-wave parametric amplification in transparent bulk Kerr medium, Opt. Express 15, 11126-11132 (2007).

[14] H. Valtna, G. Tamošauskas, A. Dubietis, and A. Piskarskas, High-energy broadband four-wave optical parametric amplification in bulk fused silica, Opt. Lett. 33, 971-973 (2008).

\title{
NAŠUS TREČIOSIOS HARMONIKOS GENERAVIMAS NAUDOJANT KETURBANGI SKIRTUMINIO DAŽNIO MAIŠYMĄ LYDYTAME KVARCE
}

\author{
J. Darginavičius, D. Majus, G. Tamošauskas, A. Dubietis \\ Vilniaus universitetas, Vilnius, Lietuva
}

\section{Santrauka}

Pademonstruotas efektyvus 1 ps trukmès šviesos impulsų trečiosios harmonikos generavimas, naudojant faziškai sinchronizuota keturbangi skirtuminio dažnio maišymą skaidrioje izotropinėje kietakūnèje terpejje. Taikant nekolinearu fazinị sinchronizmą, cilind- rini pluoštu fokusavimą bei impulso fronto pokrypi, $3 \mathrm{~mm}$ storio lydyto kvarco plokštelejje ties $351 \mathrm{~nm}$ bangos ilgiu generuoti trečiosios harmonikos impulsai, kurių energija siekè $230 \mu \mathrm{J}$, o energijos keitimo efektyvumas $-15 \%$. 